\title{
Co-authorship of Joint Utterances in Japanese
}

Yasuko Obana

YOBANA@KWANSEI.AC.JP

School of Science and Technology,

Kwansei Gakuin University

2-1, Gakuen, Sanda City, Hyogo Prefecture

Japan, 669-1337

Michael Haugh

School of Languages and Linguistics, Nathan Campus, Griffith University, Nathan, QLD 4111, Australia

M.HAUGH@GRIFFITH.EDU.AU

Editor: Raquel Fernández

\begin{abstract}
The paper introduces a type of joint utterance construction in Japanese, in which two independent sentential-level units are amalgamated, which has hitherto received little attention in the literature. Unlike traditional joint utterance construction where one speaker maintains authority over the syntactic structure of the forthcoming continuation and the other accedes to this, thereby constituting a single TCU (turn constructional unit), our examples demonstrate that both speakers can have authority over the syntactic design of joint utterances. We call such collaborative utterances 'co-authored joint utterances' in this paper.

The uniqueness of co-authored joint utterances lies in their syntactic architecture. While syntactic and semantic continuity are successfully achieved in constructing co-authored joint utterances, they represent a co-joined structure in which two sentential-level units are involved with their shared part constituting a point of amalgamation.

In analysing co-authored joint utterances, we examine how they can be treated in relation to the distinction between TCU (Turn Constructional Unit) continuation and new TCUs. Due to the particularities of the syntactic architecture of co-authored joint utterances, their existence raises questions about the way in which this distinction is currently operationalised, because despite being syntactically an incremental continuation, and so seemingly a TCU continuation, the co-authored joint utterance implements an action beyond what was initially instantiated by the antecedent of that joint utterance, and so arguably constitutes a new TCU.
\end{abstract}

Key words: joint utterance, Japanese, co-authorship, TCU

\section{Introduction}

The present paper introduces a type of joint utterances in Japanese, in which two separate 'sentential-level units' ${ }^{1}$ are amalgamated by sharing a part of each component. Joint utterances traditionally refer to "a domain of practices by which a speaker produces an utterance that is designed to grammatically continue ... an ongoing utterance initiated by another speaker"

\footnotetext{
${ }^{1}$ Although the term 'sentence' is not normally associated with spoken discourse, we use the term, 'sentential-level unit', in this paper when focusing on the syntactic analysis of a component that consists of a 'subject and predicate'. This is because many examples of co-authored joint utterances contain two 'sentential-level' units, which is quite distinct from the structure of traditional joint utterances. 
(Hayashi, 2003: 1). According to such accounts, the first speaker controls the syntactic construction and the second speaker completes it by adding modifiers or subordinate clauses, or by taking over and expanding upon the first speaker's (unfinished) utterance. However, there is another type of joint utterance that has previously received little attention, in which both speakers equally control the syntactic structure of their own utterance. We call this phenomenon a 'coauthored joint utterance' in this paper.

This paper also examines the applicability of the distinction between TCU (turn construction unit) continuation and new TCUs in relation to co-authored joint utterances. While the current distinction between TCU continuation and new TCUs grew out of Schegloff's (1996) initial claims about "increments" in English talk-in-interaction (e.g., Couper-Kuhlen \& Ono, 2007; Ford, et al., 2002; Ono \& Couper-Kuhlen, 2007), its validity has since come into question (CouperKuhlen, 2012; Krekoski, 2012; cf. Sidnell, 2012). Drawing from our analysis of co-authored joint utterances, we build on these claims in suggesting that while the distinction between TCU continuation and new TCUs may indeed serve as a resource for participants in many instances, it is not a distinction that is necessarily defensible across all cases of incremental expansion of prior units by other speakers.

Our main argument, in brief, is as follows. In the structure of co-authored joint utterances, since the second speaker's contribution is latched onto or "parasitic" on the antecedent, it appears to constitute an "other continuation" of the prior speaker's turn construction unit (TCU) (Sidnell, 2012: 316). However, the resultant joint utterance implements an action that is distinct from that initially accomplished through the antecedent, and so, in this respect, it also constitutes a new TCU (Ford \& Thompson, 1996; Schegloff, 1996). Due to these characteristics, the existence of these co-authored joint utterances raise questions about the way in which the distinction between TCU continuation and new TCUs is currently operationalised in conversational analysis (CA). As Couper-Kuhlen (2012: 276) points out, this distinction is primarily a grammatical one given it "hinges on whether the material added after a point of possible completion is syntactically dependent on the prior unit or syntactically independent from it", despite TCUs themselves now generally being defined in terms of "pragmatic or action projection" (Fox, et al., 2013: 732). However, on that account, a co-authored joint utterance seems to involve an instance of new TCU (given it implements an action distinct from that accomplished by the antecedent), on the one hand, yet, on the other hand, it is accomplished through what appears to be an instance of a TCU continuation (given that extension is recognisably syntactically dependent on the antecedent). The distinction between TCU continuation and new TCUs, therefore, appears difficult to operationalise in the case of co-authored joint utterances due to the particularities of the way in which they are constructed.

\section{Co-construction and joint utterances}

In this section, we briefly discuss previous studies of joint utterances, and also examine the semantic contributions to the construction of these utterances.

\subsection{Previous studies of joint utterances}

Joint utterances can be treated as a particular type of 'co-construction' following initial work by Jacoby and Ochs (1995). Co-construction involves all sorts of conversational phenomena in which participants jointly create a continuous flow of talk-in-interaction. However, in this paper, we focus on "the co-construction of syntactic units, namely, practices by which participants ... 
complete a sentence-in-progress started by another participant" (Hayashi \& Mori, 1998: 77). In other words, we focus on what is often loosely called a 'sentence', given it is basically comprised of subject and predicate (see footnote 1), in cases where two or more participants incrementally take turns in its construction. A joint utterance is thus a single syntactic unit of talk that is collaboratively produced.

Joint utterances were first noted by Sacks (1992a, 1992b), and since then the topic has attracted increasing attention (e.g., Bolden, 2003; Haugh, 2010; Hayashi, 1999, 2003; Hayashi \& Mori, 1998; Kim, 1999; Lerner, 1991, 1996; Lerner \& Takagi, 1999; Liddicoat, 2004; Local, 2005; Ono \& Thompson, 1996; Rühlemann, 2007; Sidnell, 2012; Szczepek, 2006; Tanaka, 1999, 2000). They are generally divided into two broad types: completions and expansions. Completion types encompass instances where "the next speaker completes a syntactic unit that the first speaker has left incomplete" (Rühlemann, 2007: 100), or more specifically, "a practice whereby a participant produces an utterance that is grammatically fitted to the ongoing trajectory of another participant's utterance-in-progress and which brings that other participant's utterance to completion" (Hayashi, 2003: 25). Expansion types of joint utterance, on the other hand, are defined as instances where "the first speaker articulates an utterance that is syntactically complete and the next speaker expands the first speaker's utterance into a longer syntactic unit" (Rühlemann, 2007: 100), by adding a subordinate clause, a prepositional phrase or an adverbial (phrase). Therefore, whether joint utterances are completions or expansions, the first speaker controls the syntactic architecture of the incipient joint utterance and the second speaker maintains it.

In the following excerpt, Rühlemann (2007: 100) provides examples of joint utterances that arise through both completion and expansion (the arrows here point to the continuations by the second speaker of the antecedent [Howes, et al., 2011: 287] produced by the prior speaker, either through completion or expansion).

\section{(1) British National Corpus: KBP2506}

1. Nina: No, they die down ( )

2. Clarence: Mm. Mm.

3. Nina: most of the ones that we brought seem to have erm

$\rightarrow$ 4. Clarence: Survived.

$\rightarrow$ 5. Nina: Survived. Which I'm glad.

6. Clarence: $\mathrm{Mm} . \mathrm{Mm}$.

In (1), in response to Nina indicating through the hesitation token erm that she is struggling to find the right word (line 3), Clarence completes the utterance begun by Nina in the subsequent turn (line 4). This constitutes an example of a completion joint utterance. Nina then further expands upon the previously co-constructed utterance with the addition of a relative clause which I'm glad (line 5), which constitutes an instance of an expansion joint utterance. In other words, through both completion and expansion the two speakers can be seen to be co-constructing a single, complex syntactic unit (Rühlemann, 2007: 101).

\subsection{Semantic contributions to constructing joint utterances}

It should be noted that even if a joint utterance is successfully created, the second speaker's prediction of the projected trajectory that can be inferred from the antecedent may or may not be 
treated as intended by the first speaker (or what is retrospectively claimed to have been intended). In extreme cases, joint utterances do not have to involve such a prediction, and yet can be successfully constituted by two (or more) participants. In this sub-section, we examine the semantic contributions made by participants in constructing a joint utterance.

In the case of example (1) above, Clarence's use of 'survived' appears to match the word Nina was searching for because Nina repeated the same word just after Clarence uttered it. However, in example (2), Izumi's completion of Tomoko's utterance in line 2 is apparently not what Tomoko was originally going to express (or was in the process of thinking of what to say) given the way she responds to it; nonetheless, Izumi's utterance turns out to be more appropriate as Tomoko accepts it ( $a$, soo $k a=$ 'oh, that's it.'). ${ }^{2}$

(2) 1 Tomoko: nanka Higashino Keigo no nandemo=

somehow of everything

"Somehow, Higashino Keigo's (books) all..."

$\longrightarrow 2$ Izumi: =hitto shi te masu mon ne. success do TE Polite MD MD "(are a) great success, aren't they?"

3 Tomoko: a soo ka. oh so MD

"Oh, that's it (I see)."

The two interactants, Tomoko and Izumi, have been talking about recent popular culture, ranging from TV programmes to novels. Higashino Keigo is a mystery writer, who is one of the most popular novelists in recent years. In this excerpt, we find a continuation of talk about his novels, in which Tomoko seemingly intended to comment on his novels. We do not know what Tomoko originally intended to say. However, what can be observed here is that Izumi takes over Tomoko's utterance, and syntactically completes it. Izumi's utterance was apparently not what Tomoko originally intended to say due to Tomoko's subsequent utterance, $A$, sooka (Oh, I see), which shows her surprise. Nonetheless, Tomoko appears to accept Izumi's continuation.

Hayashi (2003: 173-204) discusses this kind of semantic redirection steered by the second speaker in completing a joint utterance. For example,

\footnotetext{
${ }^{2}$ Examples (2) and (8) (12) discussed in this paper are from our own data, which consists of 18 interviews (each lasting 20 minutes) and two business meetings (each lasting 30 minutes), transcribed using a basic set of conventions that are listed at the end of this paper. These examples are transcribed to a level sufficient for showing how joint utterances are created from a syntactic perspective, given our focus is primarily on the syntactic architecture of joint utterances, but not, we concede, to a level sufficient for a full multimodal or CA investigation of how participants interactionally achieve co-constructions. The latter is the focus of a number of previous studies, some of which are cited near the beginning of Section 2.1. Our contention is that those kinds of interactional analyses of co-constructions can be usefully informed by closer consideration of their syntactic architecture, including cases of co-authored joint utterances, which we discuss in this paper.
} 
(3) 1 Asami: uun (ikkai) unto ne: ima uh-uh once uhm FP now "Uh-uh, (once), let's see, now..."

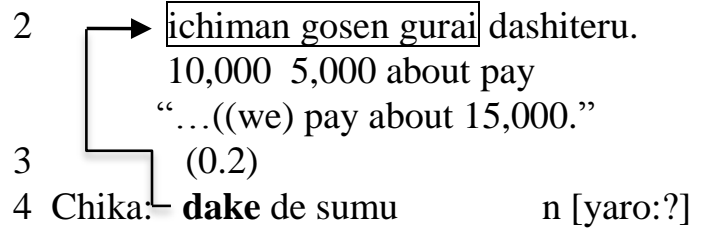

(Example (4) in Hayashi, 2003: 177)

In example (3), dake (only) in line 4 of Chika's utterance, which is termed an "utterance-initial postposition" by Hayashi, is grammatically latched onto the phrase, ichiman gosen gurai (around 15,000 yen), in line 2 of Asami's utterance. By providing further examples of utterance-initial postpositions, Hayashi asserts that "the availability of particular grammatical resources ... allows participants to accomplish a particular type of action, i.e., redirection of the trajectory of an utterance, in a particular, perhaps language-specific way" (Hayashi, 2003: 173).

Although there may be questions on whether this kind of grammatical latching can actually be strictly speaking categorised as a joint utterance ${ }^{3}$ since the utterances in lines 2 and 4 are not syntactically connected, but rather only a part inside the utterance in line 2 is taken as zero in line 4 , it does indeed constitute a type of co-construction in a broader sense (but not a joint utterance in a strict sense). However, putting aside such definitional intricacies, our primary claim here is that joint utterances do not have to be anticipatorily constructed, but the second speaker has free choice as to where his/her semantic contribution should steer the continuation of the prior utterance.

More extreme examples of joint utterances are cited by Gregoromichelaki, et al. (2011), which they call "hostile continuations", on the basis of which they assert that "incremental comprehension cannot be based primarily on guessing speaker intention" (Gregoromichelaki, et al., 2011: 209). For example:

(4) [Morse, BBC radio 7]

Morse: in any case the question was

Suspect: a VERY good question inspector

(Gregoromichelaki, et al., 2011: 208)

(5) (A and B arguing:)

A: In fact what this shows is

B: that you are an idiot.

(Gregoromichelaki, et al., 2011: 209)

\footnotetext{
${ }^{3}$ Hayashi (2003: 173) calls this type of construction "a particular utterance design in joint utterance construction".
} 
Obviously, the completions in both examples (4) and (5) diverge from the first speaker's probable intention, thereby showing the second speaker's freedom to create his/her own continuation. In fact, the second speaker semantically controls the joint utterance by bringing a hostile effect into the interaction. Therefore, the second speaker does not have to predict the first speaker's intention, and yet through syntactic continuation can co-construct a joint utterance. Such hostile continuations demonstrate that "it is not obvious why ... the addressee has to have guessed the original speaker's (propositional) intention/plan before they offer their continuations" (Gregoromichelaki, et al., 2011: 209). At the same time, "speaker intentions need not be fullyformed before production", thus, "as long as the speaker is licensed to operate with partial structures, they can start an utterance without a fully formed intention/plan as to how it will develop" (ibid, 209).

We agree with Gregoromichelaki, et al.'s (2011: 226) view that "speakers do not have to be modelled as having fully-formed messages". Instead, utterances can be expanded incrementally as the conversation proceeds, and "as long as what emerges as the eventual joint content is some compatible extension of the original speaker's goal tree (message), it may be accepted as sufficient for the purposes to hand" (ibid, 226). Likewise, the cases of co-authored joint utterances we examine here are not always the result of prediction by the second speaker of the first speaker's putative intention, but can be the result of the second speaker's own agentive continuation of the antecedent. In other words, the second speaker is not bound down by inferences about the first speaker's original putative intentions or the action trajectory implemented by the first speaker's utterance, but can go beyond it to achieve a distinct action trajectory or even a new direction of activity in the given discourse.

What this sub-section has illustrated is that joint utterances do not necessarily require the initial semantic direction or action trajectory implemented by the first speaker to be maintained by the second speaker. Thus, what makes a particular utterance a joint utterance is syntactic continuity passed on from one speaker to another. In most studies to date, joint utterances are typically taken to involve the completion of an ostensibly unfinished unit of talk with further talk that is syntactically continuous, but because the subsequent completion is made by more than one participant, the term 'joint utterance' has been given, in order to distinguish it from other types of co-constructed utterances. ${ }^{4}$ Likewise, the examples of co-authored joint utterances introduced in this paper apparently preserve syntactic continuity. However, unlike examples of completion or expansion joint utterances, which constitute a single sentential-level unit, co-authored joint utterances are derived from two independent sentential-level units, a part of which is amalgamated, thereby creating a unique syntactic design.

\section{Co-authored joint utterance constructions}

In this section, we analyse examples of co-authored joint utterances, and by examining how they are constructed, we highlight significant features of such utterances, as well as discuss how they differ from other types of co-constructions.

\subsection{Analysis of co-authored joint utterances}

\footnotetext{
${ }^{4}$ Another type of joint utterance that has received attention is what are termed expansion joint utterances. However, both completion and expansion types of joint utterance constitute a single 'sentential-level unit'.
} 
Examples of joint utterances in previous studies generally refer to either completions or expansions, in which it is the first speaker who initiates and controls the syntactic structure of the joint utterance while the second speaker maintains it by adding further units through either completion or expansion of the antecedent. Those examples present a (completed) simple sentential-level unit, which has been jointly constructed by participants. In this section, however, we discuss co-authored joint utterances in Japanese, in which two syntactically independent, sentential-level units are partially amalgamated, and tied in such a way that the resultant structure presents unique structural intricacy that goes beyond the scope of most extant accounts of grammar. ${ }^{5}$ In the case of co-authored joint utterances, therefore, both speakers have authority over the syntactic design (as well as the semantic direction) of the resultant utterance.

In example (6), for instance, we can observe how the two participants take turns to construct a continuous joint utterance, in which two independent sentential-level units are integrated into one through sharing the same object.

(6) $\left(\mathrm{BTS}^{6}\right)$

1 F02: minna kai-tai-hoodai

everybody buy-want-freely

"Everybody bought ( ) freely"

2 F01: fudan zettai kawa-nai mono toka

usually never buy-not things such as

"things like what (they) usually never buy"

3 F02: gamanshi-te-iru mono o

resist-TE-Prog thing ACC

"things (they) had resisted (to buy)"

4 F01: zettai ire-te-ta yo, are.

certainly put-TE-Past MD indeed

"(they) surely put (such things into the basket), indeed."

Two female friends refer to their sports training camp and start talking about how generous their coach was because he gave 10,000 yen for the students to buy whatever they liked in a shop. This excerpt describes how they went shopping.

The four utterances in example (6) are all connected syntactically to create a co-authored joint utterance. The syntactic architecture of these interconnected four utterances is shown in two alternative ways in Figure 1 below.

\footnotetext{
${ }^{5}$ However, Dynamic Syntax (Cann, et al., 2005; Kempson, et al., 2001) and Poesio and Riser's (2010) Dialogue model represent important exceptions to this trend, as does recent work in interactional linguistics (e.g., Auer, 2009; Laury \& Ono, 2014).

${ }^{6}$ BTS refers to data taken from the corpus of spoken Japanese compiled by Mayumi Usami and her team at Tokyo University of Foreign Studies, which is transcribed using the 'Basic Transcription System' (BTS). This excerpt and the other examples indicated as taken from that corpus are presented as they were transcribed using the BTS, although we use the romanised form of Japanese in this paper for the sake of reader accessibility. How Dynamic Syntax (Cann, et al., 2005; Kempson, et al., 2001), which Gregoromichelaki, et al. (2011) argue is well equipped to handle joint utterances more generally, would treat co-authored joint utterances is an intriguing question, but lies outside the scope of this current paper.
} 
1 F02: Subject Predicate 1

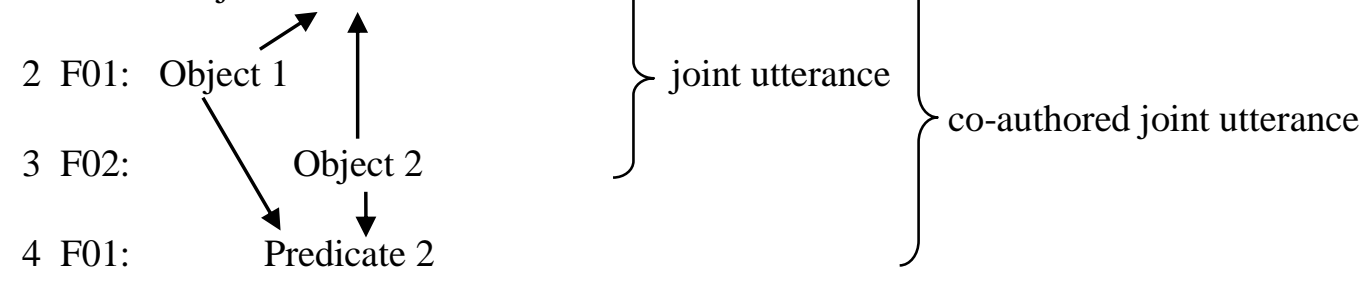

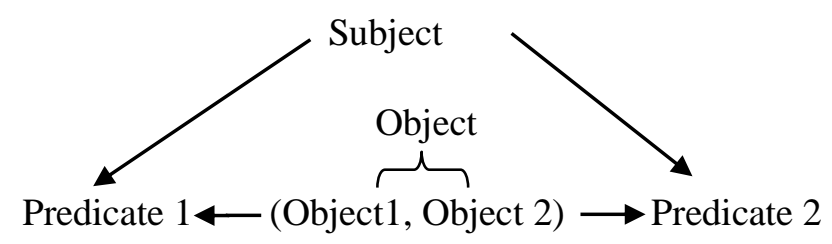

(NB: $\mathrm{a} \longrightarrow \mathrm{b}=$ 'a' modifies 'b')

Figure 1. The syntactic architecture of the utterances in example (6).

Figure 1 illustrates that F02's first utterance (line 1) consists of a subject and predicate (Predicate 1), and that F01's first utterance (line 2) consists of an object (Object 1), while F02's second utterance (line 3) constitutes an additional object (Object 2), which apparently functions as a postpositioned insertion into the syntactic structure in line 1, namely as the argument of Predicate 1. This means that the utterances in lines 1 to 3 constitute a traditional joint utterance with the objects as increments (Schegloff, 1996: 73f). However, the utterance in line 4 brings up another predicate (Predicate 2), which equally takes the same object (Objects $1+2$ ) as its argument (but realised as zero). This means that the object here is shared between Predicates 1 and 2. The subject (minna, 'everybody'), which appears in line 1, persists throughout excerpt (6) and is thus omitted in line 4.

The two participants, by adding utterances incrementally, successfully construct a co-authored joint utterance in which the two sentential-level units (one unit comprising the utterances in lines 1 to 3 and the other in lines 2 to 4 ) are merged into one component with the argument (i.e., the object in lines 2 and 3) being shared between those two sentential-level units. This is not a mere completion, where the second speaker takes over finishing off the first speaker's utterance. Nor is it an expansion, ${ }^{7}$ whereby the second speaker adds peripheral units to complement the first speaker's utterance. While both speakers control their own syntactic design, two distinct units are amalgamated into one continuous component. These continuous turns also achieve wellcoordinated semantic continuity.

Co-authored joint utterances such as example (6), which show the participants' relayed participation, ${ }^{8}$ are most likely to occur when the participants are in close accord, appearing to share the same (or similar) feelings about their common experiences. In a similar way, example (7) illustrates an instance where the two female participants agree that they as seniors (senpai) in their club prefer not to be involved in preparing for the forthcoming Christmas party, and wish

\footnotetext{
${ }^{7}$ In example (6), Objects 1 and 2 are in coordination, and both fill in the object position.

${ }^{8}$ We note in passing that this kind of relayed participation may be parasitic in part on the phenomenon of "clause chaining" that single speakers have been observed to accomplish in Japanese conversations (see Laury and Ono, 2014: 571 , and references therein).
} 
that they could just turn up at the party and pay the fee, although in reality senpai are obliged to work hard to prepare for the party. Their shared feelings create a kind of relayed conversation, in which a co-authored joint utterance is incrementally accomplished.

(7) (BTS)

1 F14: senpai toshite motehayasa-re-tai

senior as hail-Pass-want

"(I) want to be hailed as a senpai"

2 aa, senpaai toka it-te, mujookenni.

oh senpai etc. say-TE unconditionally

"(they) say unconditionally such as 'wow, senpai'."

3 F13: ki-te-kure-ta-n-desu kaa toka it-te

come-TE-receive-Past-Nomi-Polite MD like say-TE

"like (they) say, '(you) have kindly come (to the party)."

4 F14: ne

MD

"Indeed"

5 F13: irasshai toka it-te

welcome like say-TE

"(They) say such as 'welcome'."

6 F14: soo soo soo soo

yep yep yep yep

"Yep, yep, yep, yep."

7 F13: okane atsume-rare-te-mitai

money collect-Pass-TE-try(want)

"(I) once want to have (my) money collected (that way)."

The units in lines 1 and 2 constitute an inverted sentence (with the unit in line 1 as a main clause), and the utterances in lines 3 and 5 are an expansion added to the main clause in line 1, which creates a traditional, expansion-type joint utterance. However, the subordinate clauses in lines 2, 3 and 5 at the same time modify the main clause in line 7 . This means that the two sentential-level units (one unit comprising the utterances in lines 1 3 and 5 and the other in lines 2, 3, 5 and 7) are amalgamated with the subordinate clauses in lines 2, 3 and 5 being shared, and so form a coauthored joint utterance. This is illustrated in Figure 2 below.
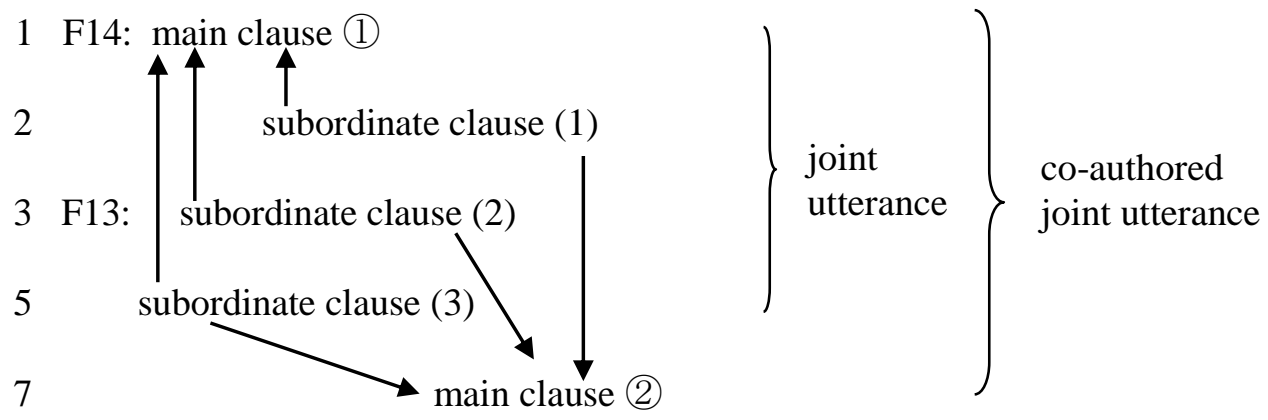

Figure 2. The syntactic relations between the utterances to construct joint utterances 
Figure 2 indicates that the two main clauses, (1) and (2), are linked with the three subordinate clauses, (1) (3); the subordinate clauses thus belong to both of the main clauses.

Semantically, the two participants keep the same line of thought by adding information incrementally, creating a kind of relayed conversation. Syntactically, the second speaker (F13) initially contributed to an expansion of F14's utterance (a typical example of an "increment": Schegloff, 1996: 73f) in lines 3 and 5, thereby creating a traditional joint utterance. However, the main clause in line 7 reframes the whole discourse into a co-authored joint utterance where the two main clauses, (1) and (2), are involved and fused with the antecedent subordinate clauses, (1) $\sim(3)$.

One might argue that co-authored joint utterances can be constructed only when the participants know exactly what they are talking about due to their shared experience as indicated in examples (6) and (7). However, example (8) shows that shared knowledge (or experience) is not sine qua non to the achievement of co-authored joint utterances. This is because they invariably arise through the grammatical conversion of a prior syntactic unit, and so involve more than simply the sharing of common experiences or stances.

Example (8) is taken from an interview which Tomoko (who is a student) conducted with another student, Rie. They knew each other by name, and so Tomoko first asked where Rie is living and then, Rie asked where Tomoko is living. Tomoko said the name of a place, but apparently Rie did not hear it clearly. The excerpt below follows on from this.

(8) 1 Tomoko: wakaru? [Hooryuuji] Hooryuuji= understand Horyuji

$$
\text { "(Did you) get it? Horyuji, Horyuji) }
$$

2 Rie: [hai hai]

"Yes, yes"

3 =no chikaku desu ka? of nearby Polite Q "(Are you living) near (Horyuji Temple)?"

4 Tomoko: EKI. station "(near) the (Horyuji) Station"

5 Rie: metcha kakkoii desu ne. very cool polite MD "Very cool, indeed."

6 Tomoko: (.) tte iwa reru yooninat ta otona ninat te. Quote say Pass become Past adult become TE "(So I am) told since (I) became grown-up."

The utterances in lines 1 and 3 are syntactically merged into a continuous unit as shown in Figure 3. 
1 Tomoko: Hooryuuji. (one-word completed utterance)

3 Rie:

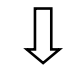

$\emptyset$

no chikaku desu ka.

(noun)

Figure 3. The conversion of a one-word complete utterance into a noun in another utterance

Tomoko answered that she is living in Horyuji. Horyuji can refer to the name of a place (or that of a railway station) in Nara, but at the same time it is also the name of a historically famous temple. Tomoko meant the former, as it turns out, but Rie initially interpreted the reference as the latter. This is why Rie asked whether Tomoko is living near the temple (line 3). The two utterances constitute a co-authored joint utterance, in which Tomoko's predicate noun (Hooryuuji) is shared between the two independent utterances. Tomoko completes her utterance by producing one word with a descending tone, but this utterance is taken advantage of and utilized as a noun (but empty in the syntactic slot) in Rie's subsequent utterance (line 3), which combines with a possessive particle, no, leading to the creation of a completely new sententiallevel structure (i.e., an interrogative). Unlike examples (6) and (7), which are semantically driven in the same direction by adding further information step-by-step in a kind of 'relay', example (8) involves a different orientation in the case of the continuation of the antecedent of the joint utterance. Although Rie's utterance does not deviate from trajectory at the macro level of discourse (i.e., talking about where they are currently living), it redirects the action trajectory of the prior utterance by creating a question to Tomoko through a continuation of Tomoko's prior utterance. Tomoko's utterance in line 1 is framed as an answer to a prior question raised by Rie, but is reframed as a part of a question by Rie through a syntactic continuation of Tomoko's utterance.

The utterances in lines 5 and 6 constitute another co-authored joint utterance, whose syntactic relations are shown in Figure 4.

5 Rie: metcha kakkoii desu ne (completed sentential-level unit)

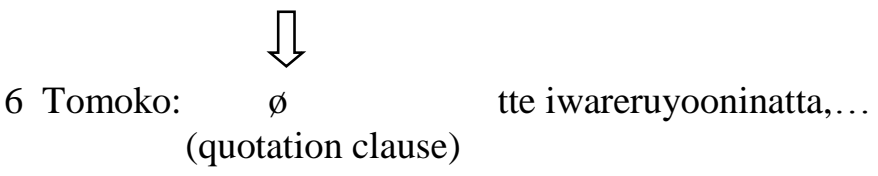

Figure 4. Quotation conversion

Rie commented that Tomoko's place is really cool because Horyuji is historically famous. Rie's utterance is completed with the mood marker, ne (indeed), which indicates a possible end of Rie's turn. However, Tomoko incorporates this utterance into her new utterance, by converting the former into a quotation clause in Tomoko's subsequent utterance. Tomoko replied that since she entered adulthood, she has often received such comments. Syntactically, the quotation clause is omitted (i.e. a zero anaphor indicated as ' $\varnothing$ ' in Figure 4), and in order to achieve syntactic continuation, Tomoko's utterance employs anastrophe by inverting the two clauses, otonaninatte ('since I was grown up') and $\phi$ tte iwareruyooninatta ('so [I] have been told'). Semantically, Tomoko shifts Rie's frame into a different one, that is, from an assessment into a quotation about 
which Tomoko takes a particular stance. Yet due to the syntactic continuity, Tomoko claims equal authorship with Rie, which gives rise to a co-authored joint utterance.

This kind of 'quotation conversion' often occurs in our data, especially in business meetings. For example:

(9) Fukuda: kyoo juuni kuru?

today within come

"(Does it) come sometime today?"

(.)

Doi: toyuu yotei desu.

Quote schedule Polite

"(that it will come today) has been scheduled."

(10)Fukuda: sore moo tyuumon ni dashi ta?

that already order to dispatch Past

"(Did they) order that already?"

(.)

Endo: toyuu hookoku o uke te i masu

Quote report ACC receive TE Prog Polite

"(I) received a report (that it has already been ordered)."

Both (9) and (10) are excerpts from a business meeting in which Fukuda as a senior person checks tasks each junior member was supposed to complete. In both examples, the first speaker's completed utterance with a raising tone, here framing it as a question, is converted into a quotation clause through the continuation by the second speaker. This means that while two sentential-level units are merged into one component, both speakers not only control their own syntactic design but also proffer two different action trajectories, that is, a question and an answer.

It should be noted that this conversion is possible only when the first speaker's sentence structure is affirmative with a raising tone. If the first speaker's structure is formulated in an interrogative form with a question marker, $k a$ or no, the second speaker cannot create a joint utterance. This means that in spite of the raising tone in the first speaker's utterance, which forms a question, the second speaker focuses only on the syntactic trajectory and adds further units, which serve as an answer to the first speaker's enquiry.

In sum, joint utterance construction has traditionally been regarded as allowing "the original speaker to maintain his/her authority over a turn even when completed by another" (Lerner, 2004: 225). However, the examples of co-authored joint utterances that we have discussed indicate that both speakers contribute talk with different syntactic designs and yet this constitutes a syntactically continuous construction by sharing a part across the two independent sententiallevel units. A syntactically shared part between the two sentential-level units modifies the head of each utterance as shown in example (6), modifies the main clause as shown in (7), or is grammatically converted into a different unit in the second speaker's continuation as shown in examples (8) to (10).

It may seem in our analysis thus far that we have emphasised syntactic aspects of co-authored joint utterances at the expense of a consideration of the role of semantic links in the construction of co-authored joint utterances. Indeed, co-authored joint utterances cannot be constructed without semantic considerations, and the semantic contribution of utterances is important to 
consider in analysing how the units in the co-authored joint utterance are related to one another. For example, the co-authored joint utterances in examples (6) and (7) constitute a continuous relay as if one person were uttering a single utterance, even though the utterance arises over multiple turns by the two participants. In examples (8) to (10), both speakers exhibit their own authorship in interaction, and semantic divergence occurs because each participant projects their own stance with respect to the action trajectory in question. However, it is rather the unique syntactic architecture (i.e., syntactic amalgamation) of co-authored joint utterances that distinguishes them from other types of co-construction. Co-constructions involve all sorts of cases in which the participants jointly create a continuous flow of talk-in-action. They may reveal only fragments of utterances, offer only semantic connections between utterances, and/or consist of independent utterances which nonetheless achieve a semantically relevant flow. On the other hand, co-authored joint utterances, which we argue constitute yet another different type of coconstruction, exhibit the syntactic amalgamation of two independent sentential-units, and the resultant construction is so tightly-entwined that it is arguably structurally inseparable.

\title{
3.2 Anaphoric relations in co-authored joint utterances
}

The main reason why co-authored joint utterances are attainable in Japanese is that the Japanese language allows ellipsis to occur in all sorts of syntactic positions. This is particularly conspicuous in conversation. For instance, the utterance in line 1 of example (6) can stand on its own with its object omitted although the verb ( $k a u=$ 'to buy') is potentially transitive. In examples (8) to (10), the second speaker takes over the first speaker's utterance and utilizes it syntactically as zero in the former's continuation of the first speaker's utterance, and yet it is naturally accepted in conversation. As a matter of fact, the participants take advantage of the omitted units, which come into play in building up a co-authored joint utterance. Because of this, one may wonder if co-authored joint utterances can be analysed with reference to anaphoric relations alone. If not, as we are arguing, then what distinguishes co-authored joint utterances from other elliptic co-constructions?

To further consider this question, let us look at example (11), which appears at first glance to be a syntactic continuation that is collaboratively constituted.

\section{(11) (BTS)}

\author{
F02: sugokat-ta ne are wa. \\ terrific-Past MD that Top \\ "Terrific, that was." \\ F01: omoshirokat-ta, un. \\ fun-Past hmm \\ "(That was) interesting, indeed."
}

This excerpt follows on from example (6), and involves the participants commenting on the experiences they shared in the camp. F02's utterance is syntactically scrambled between subject and predicate, and completed with a subject. F01's utterance starts without a subject, but it is evident that the subject is the same as that of F02's utterance. The subject, are wa ('that'), appears to be the merging point between the two utterances. From this perspective, example (11) can be treated as an instance of a co-authored joint utterance. However, since are wa does not receive any grammatical conversion but remains as the same subject in F01's utterance, it is also 
possible to assume that the subject in F01's utterance is not a merging point, but merely a case of ellipsis. That is, two separate utterances are displayed, but because the subject has already occurred in F02's utterance, it is omitted for the sake of establishing topic continuity ${ }^{9}$ (Givón 1983; Hinds, 1978, 1982, 1983, 1984). Therefore, inverted structures like F02's utterance in example (11) are somewhat more controversial as candidate instances of co-authored joint utterances. It is difficult to conclusively determine whether it should be treated as a co-authored joint utterance or as two separate, distinct units with an anaphoric relation.

To avoid such controversies, it is possible to limit the scope of co-authored joint utterances to those which exhibit grammatical conversion at the merging point (except relay-like structures as shown in examples (6) and (7); this will be discussed below). For instance, in example (8), Hooryuuji (Horyuji Temple) as a one-word completed utterance in line 1 is converted into a simple noun in a noun phrase in line 3. In example (9), Fukuda's utterance, which is a completed utterance as a question, is converted into a subordinate clause in Doi's utterance. On the other hand, example (11) does not display such a grammatical conversion since the same unit is merely shared between the two utterances. We thus regard this phenomenon as a simple case of ellipsis, as shown in Figure 5.

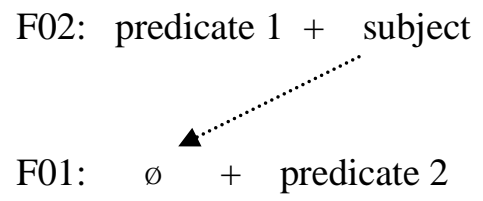

Figure 5. The subject realised as a zero anaphor in the subsequent utterance

Grammatical conversion may be a solution to distinguish co-authored joint utterances from other elliptic cases like example (11). However, grammatical conversion does not occur in examples (6) and (7) although we claim these examples should be considered co-authored joint utterances. It might therefore be argued that examples (6) and (7) can be explained as forms of ellipsis.

Indeed, we admit that examples (6) and (7) involve anaphoric relations with zero units shared between the utterances, and co-authored joint utterances are obtained because they take advantage of the phenomena of ellipsis in Japanese. However, unlike other utterances bearing ellipsis, coauthored joint utterances bring in syntactic continuity which builds up a unique structure: the merging of two independent sentential-units. This unorthodox syntactic architecture distinguishes co-authored joint utterances from other zero-anaphoric utterances. In other words, while elliptic utterances in general only indicate how zeros refer back to relevant antecedents, co-authored joint utterances go further and exploit zeros to create an amalgamated syntactic continuation.

\section{Joint utterances and the role of syntax in turn continuation}

Turn constructional units (TCUs) are one of major features often highlighted to explain turntaking systems in CA. Sacks, et al. (1974) originally defined a TCU as a recognisably complete

\footnotetext{
${ }^{9}$ Zero anaphora is considered to be on top of the coding topic accessibility. In example (11), for instance, are wa (with $w a$ as a topic marker) is assigned as a topic in the discourse, and in subsequent structures, the topic is omitted as evidence of "the unmarked form of topic continuity" (Hinds, 1983:49).
} 
unit in conversation. TCUs can be words, phrases, clauses and sentences, and participants predict through their talk where they expect a given TCU is coming to its point of possible completion. While it is not always acknowledged in CA, Sacks, et al. (1974) in fact relied heavily on syntactic constructions in conceptualising TCUs, because a new TCU (by another speaker, for example) does not start arbitrarily at any point in the previous utterance, but in the vicinity of the projectable completion point of a certain syntactically self-contained unit (Ford, et al., 1996, 2013). In this respect, traditional joint utterance construction is an interesting case because a single unit is collaboratively produced. Lerner (1991) considers joint utterance constructions to nevertheless constitute single TCUs, terming such cases a type of "compound TCU", because it "projects in its course, and prior to the onset of a final component, that a two-part unit is underway" (Lerner \& Takagi, 1999: 53). This assumption seems to be plausible because a traditional joint utterance constitutes a single syntactic unit, as well as constituting a single unit of action $^{10}$, except that it is contributed to by more than one participant.

However, the instances of co-authored joint utterances we have examined suggest that the proposed distinction between TCU continuation and new TCUs (Couper-Kuhlen \& Ono, 2007; Ford, et al., 2002; Schegloff, 1996) may be more difficult to maintain than has been assumed to date. The first issue is that co-authored joint utterances consist of two independent sentential-level units; the first component is already completed, and yet the second component continues on to attach another syntactically autonomous sentential-level unit (although taking advantage of a part of the first component). This means that the second component is not simply an "increment" (Schegloff, 1996), but constitutes another form of syntactic continuation.

The second issue is that co-authored joint utterances often involve cases where the second speaker's continuation deviates from the incipient trajectory of the first speaker's utterance, and creates a new semantically or pragmatically independent unit, and yet this is not subsequently contested or resisted by the first speaker. These observations thus cast doubt on the applicability of the distinction between TCU continuation and new TCUs in the case of co-authored joint utterances, given there is clearly syntactic continuation at play here (and so is recognisable as an instance of TCU continuation to participants), and yet the action instantiated through the joint utterance can differ from the incipient action trajectory of the antecedent (and so a co-authored joint utterance is recognisable as an instance of a new TCU to participants).

In this section, we first review previous studies on TCU continuation in further detail, and then examine whether or not the distinction between TCU continuation and new TCUs can apply to our examples of co-authored joint utterances.

\subsection{Previous studies of increments and TCU continuation}

The distinction between TCU continuations and new TCUs is closely related to the finding that in natural conversation, not all transition-relevance places (TRPs) necessarily lead to speaker

\footnotetext{
${ }^{10}$ However, if action is taken into consideration, Gregoromichelaki, et al.'s (2011) "hostile continuations" and Hayashi's (2003) "utterance-initial postpositions" discussed in Section 2.2 would be problematic as they redirect the action initiated by the prior speaker, thereby, giving rise to two units of action in the single (compound) TCU. Equally problematic are Purver et al.'s (2010) “split utterances" where speaker/addressee pronouns change as the speaker transition occurs (e.g., A: "Did you give me back" B: "your penknife?...": Purver, et al., 2010: 43), and Gregoromichelaki, et al.'s (2011) 'question-answer' type joint utterance (e.g., A: "Are you left or" B: "right handed” : Gregoromichelaki, et al., 2011: 208).
} 
change $^{11}$, a claim that is now well established in CA (Clayman, 2013; Sacks, et al., 1974; Schegloff, 1996). This is partly because utterances in conversation may contain repetitions, repairs or retrospective add-ons by the current speaker, through which a potential TRP is circumvented in spite of the projectable completion of a TCU. For example, the same speaker keeps his/her turn and continues talking after apparently having completed his/her utterance due to a particular array of syntactic and prosodic features that include compressing the TRP or bridging it through pivots (Clayman, 2013).

The term "increment" was initially used by Schegloff (1996) to refer to a syntactically extended component added to the host TCU. An increment is grammatically continuous to the prior-host TCU, and so is syntactically dependent on the host which itself stands as a complete TCU. Therefore, increments are a form of TCU continuation (Schegloff, 2001). On the other hand, a new TCU is pragmatically independent, and is claimed to not be related to the prior unit (Couper-Kuhlen, 2012: 276), although Schegloff (1996: 76) also discusses cases where recognisably new TCUs are "grammatically continuous with what preceded". Ford, et al. (2002: 16) follow suit, referring to an increment as a "non-main clause after a possible point of turn completion".

In the following example taken from two students' conversation, we can find evidence of the existence of these kinds of incremental continuations in Japanese.

(12) 1 Tomoko: watashi kekkoo yotchaun desu yo.

I quite get sick Polite MD

"I often feel sick, actually."

2 Hiroko: densha de?

train on

"on the train?"

3 Tomoko: furansu no TGV desae yotta koto aru-n desu yo.

France of TGV even get sick-Past thing exist-Nomi Polite MD

"(I) have been sick even on TGV in France."

4 Hiroko: E:::?

really

"Really?"

$\longrightarrow 5$ Tomoko: ha ha ha sonnani yurenai noni

laugh so much shake though

"(laugh) though (that train) does not rattle around so much"

In example (12), the utterance in line 5 is an incremental expansion of the utterance in line 3 which is the host component. The two participants were talking about travelling, and Tomoko told Hiroko that she does not particularly like travelling by train because she often feels sick (line 1), and she felt sick even on the TGV (line 3). She then added 'though [that train] does not rattle around so much' (line 5). Although the utterance in line 3 is a complete TCU and Hiroko's interjection enters straight after line 3 , the utterance in line 5 evidently constitutes a continuation of the TCU in line 3 due to its semantic and syntactic dependence on the TCU in line 3 . Therefore,

\footnotetext{
${ }^{11}$ For example, Selting (2000) points out that in story-telling contexts, speakers often present multiple TCUs within a single turn, (temporarily) suspending the sequential relevance of TRPs.
} 
the component in line 5 is analysable as an increment, and thus the utterances in lines 3 and 5 can be considered to be an example of TCU continuation.

In this way, then, TCU continuations have been determined mainly based on syntactic continuity, where add-ons are incremental to and syntactically dependent on the host component (e.g. Couper-Khulen \& Ono, 2007; Ford, et al., 2002; Schegloff, 1996, 2001), although CouperKhulen \& Ono (2007) and Ford, et al. (2002) also admit the importance of prosody and other pragmatic features in analysing increments.

However, the central role placed on syntax in dealing with TCU continuation has been challenged by other researchers, who take a more holistic view of TCUs and include semantic, prosodic and other pragmatic features (e.g. Auer, 2007; Ford, 2004; Ford, et al., 1996; Krekoski, 2012; Luke \& Zhang, 2007; Sidnell, 2012). Streeck and Hartge (1992) even include extralinguistic features such as gesture, gaze, facial expressions in locating TRPs.

Let us look at a few examples. Luke and Zhang (2007) go into detail in describing different types of increments, and argue that certain "insertables" in Chinese, although they appear to be syntactically incremental and thus dependent, should be considered free constituents. For example, when cai ('just', an adverb) is retrospectively added to an apparently completed component, it can syntactically be placed back into this host component. However, Luke and Zhang (2007: 622f) consider this adverb to nevertheless constitute a new TCU because the host component ends with the descending tone and a slight pause. Thus, they argue that syntactic continuation alone cannot determine whether the unit concerned is continuous to the host component (thus, constituting one continuous TCU) or distinctive from the host (thus, accounting for two TCUs). They conclude that prosody should be taken into consideration.

If prosody should be considered in distinguishing TCU continuation from new TCUs, as argued by Luke and Zhang (2007), the utterance in line 5 of example (12) above should be regarded as a new TCU because the utterance in line 3 ends with a descending tone and stands as a complete TCU in its own right, which thus allows the other speaker to enter the interaction straight after the utterance in line 3. In a similar way, Luke and Zhang's (2007) claim would undermine Krekoski's (2012) argument about increments as they would presumably consider the utterance in line 3 of example (13) to constitute a new TCU.

(13) 1 M: [suki da kara nani mo] iemasen kedo ne. like therefore nothing say:can:not but FP

"he likes it so nothing can be said"

$2 \mathrm{~K}: . . \mathrm{n}=$.

"Mhm"

3 M: ... kotchi wa.

this side WA

"(by) me (lit. '(as for) this side')"

(adapted from Krekoski, 2012: 301)

However, Krekoski's (2012) claim is that M's talk in line 3 is an increment relative to prior talk in line 1. He argues that although the utterance in line 1 ends with a descending tone and "marks clearly syntactic closure", the subsequent talk in line 3 shows that "the speaker opts to continue the previous possible completed turn with the increment" and so concludes that "line 3 represents 
an extension of the prior action, and is retrospectively oriented" (Krekoski, 2012: 301). However, according to Luke and Zhang (2007), this insertable utterance should be considered a new TCU.

One may argue that Luke and Zhang's (2007) insertable is a mere add-on (or an adjunct syntactically), whereas the add-on in line 3 of example (13) is a filler which is syntactically missing in the host (line 1). This may be a crucial point that distinguishes between increments and new TCUs, although neither Luke and Zhang (2007) nor Krekoski (2012) refer to this criterion. If this criterion is employed, what could be treated as a mere increment in line 5 in example (12) should be considered a new TCU. However, this creates further complications. According to Schegloff (2001) and Ford, et al. (2002), increments are grammatically continuous to the main (host) clause, which can be integrated into the host component; that is, increments are non-main clauses as extensions to the host clause. However, if prosody and pause are to be incorporated, as argued by Luke and Zhang (2007), the current definition of increments should be re-examined; otherwise, different scholars would present different analyses of the same phenomena when distinguishing between TCU continuation and new TCUs.

Krekoski (2012) further argues that even syntactically independent utterances can be considered continuations as a result of prosodic effects. For example,

(14) $1 \mathrm{~W}$ : ..datte tsukuru dake ja sumanai mon.

but prepare only DEWA end:NEG FP

"but (it) doesn't end only with preparing (meals),"

2 .. katazukeru mon

tidy $F P$

"(they/we/I) tidy up (the house)."

3 ... kaimono mo suru mon.

shopping also do FP

"(they/we/I) also do the shopping."

(adapted from Krekoski, 2012: 308)

Although example (14) consists of three completely independent clauses, prosodic patterns are repetitive with mon (a mood marker indicating the end of a clause) carrying the same declining tone in all utterances in lines, 1 to 3 , with a short pause in each clause. Therefore, Krekoski (2012: 309) concludes that the utterances in lines 2 and 3 are "strongly and retrospectively oriented and pragmatically bound together, and seem to be serving as a continuation of action initiated with the clause ending" in line 1. This means that Krekoski treats "prosodic coherence linking the material together" (Krekoski, 2012: 311) as an indicator of TCU continuation even if the utterances are all syntactically independent.

Krekoski's (2012) idea directly challenges the definition of increments by Schegloff (1996) because increments are supposed to be syntactically bound to the host component. According to Schegloff's definition, continuations like example (14) should not be considered increments but other types of continuation. Krekoski's assumption also appears to undermine Lerner's (2004) treatment of TCU continuation, because Lerner excludes coordinated sentences linked with and from the category of TCU continuations since each clause in the coordination is complete and could potentially achieve a distinct unit. In this respect, Lerner (2004) is apparently in line with Schegloff (1996) who places value on syntactic dependence in determining whether something constitutes a TCU continuation. However, if Krekoski's (2012) approach is employed, 
coordination should be regarded as a single TCU because it binds two clauses prosodically as well as pragmatically, and the speaker's 'intention' to continue talking after the first clause can be clearly observed.

\subsection{Co-authored joint utterances and TCU continuations}

Previous studies of TCU continuation seem to show that in spite of general acceptance of the term, its definition is not clear enough to explain many phenomena, and therefore, researchers resort to or add on different features such as prosody, epistemic values or putative speaker intentions to distinguish between TCU continuation and new TCUs. However, this has led different scholars to arriving at different conclusions, and controversy over the distinction between TCU continuation and new TCUs is still ongoing.

The examples of co-authored joint utterances examined in this paper serve to further complicate this issue because they intersect with all the potential controversies discussed in Section 4.1. First, because two sentential-level units are amalgamated in the case of co-authored joint utterances, the concept of 'increment' does not apply because increments are supposed to be syntactically dependent on the host component. Second, while both speakers implement an independent utterance with its own action trajectory and claim equal authorship, the construction as a whole is syntactically continuous. This is a new phenomenon that has not been considered to date, because studies of TCU continuation have focussed on two (or more) components in which one is dominant and the other(s) syntactically dependent. Third, in building up co-authored joint utterances, the second speaker does not necessarily maintain the same trajectory as that of the first speaker; the former has freedom to exercise control over the directionality as long as the overall flow of conversation remains intact. Finally, prosody and inferences about the first speaker's putative intentions with respect to the antecedent are often irrelevant to the second speaker's continuation, and yet the whole construction is semantically as well as syntactically continuous.

The question thus arises: is it really necessary to determine whether co-authored joint utterances involve TCU continuation or consist of two independent TCUs? We believe it is not because phenomena of co-authored joint utterances go beyond the syntactic mechanisms that are generally invoked when making this distinction. For example, let us return to consider how example (6) can be parsed.

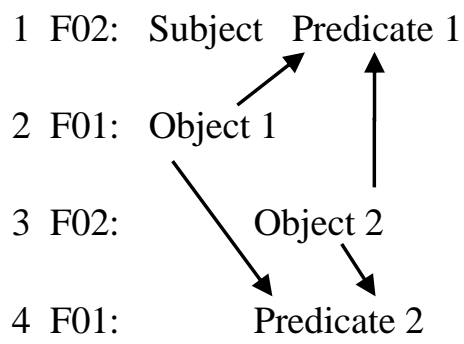

Figure 1. Example (6)

Figure 1 shows that by taking advantage of a zero argument in the object position in line 1, two speakers add on Objects 1 and 2 to Predicate 1 (of the first speaker, F02), and then the speaker, F01, in line 4, further takes advantage of these add-ons to link to a new predicate (Predicate 2). Although two sentential-level units (the utterances in lines 1 and 4 with incremental units in lines 2 and 3) are joined, there is no cut-off point syntactically; it is a symmetrically conjoined unit that 
is tightly knitted into one continuous unit. Semantically the two participants cooperate in building up continuous discourse by adding information each in turn. Originally line 1 is treated as a complete, independent clause, and therefore, the utterances in lines 2 and 3 can be considered increments. However, the utterance in line 4 is not a simple add-on but a predicate which takes advantage of Objects 1 and 2, forming another distinct sentential-level unit. Therefore, Figure 1 presents a tightly-knitted construction, which is created by amalgamating two independent sentential-level units, and yet gives rise to a semantically coherent unit. This kind of construction is not a matter of 'conversational turns' but a jointly-interlaced or fused component that cannot be parsed any longer once it is formulated in that way.

The grammatical conversions shown in examples (8) to (10) present more intricate issues. Let us look at Figure 4 again, which we have altered slightly for the subsequent discussion below.

Rie: metcha kakkoii desu ne (completed sentence)

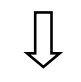

Tomoko: $\quad \varnothing$ (quotation clause)

tte iwareruyooninatta, otona ni nat-te.

(Rie: [it's] really cool.

Tomoko: [So I am] told, since [I] became grown-up.)

Figure 4. Quotation conversion

Rie comments on the place where Tomoko is living, which is uttered as a complete utterance with ne (a mood marker which projects possible completion of the turn, i.e., a TRP). However, this independent sentential-level unit is used as a quotation (clause) in Tomoko's next utterance. In that way, Rie's complete sentential-level unit is converted into a quotation clause in Tomoko's construction, although it is realised as zero in reality. Grammatical conversions are possible because Japanese is a head-final language and also allows ellipsis to occur in all sorts of syntactic slots. The first speaker's component is used as an embedded clause in the second speaker's component, and the second speaker's main clause occurs on the right-hand node in syntax, as is shown in Figure 7.

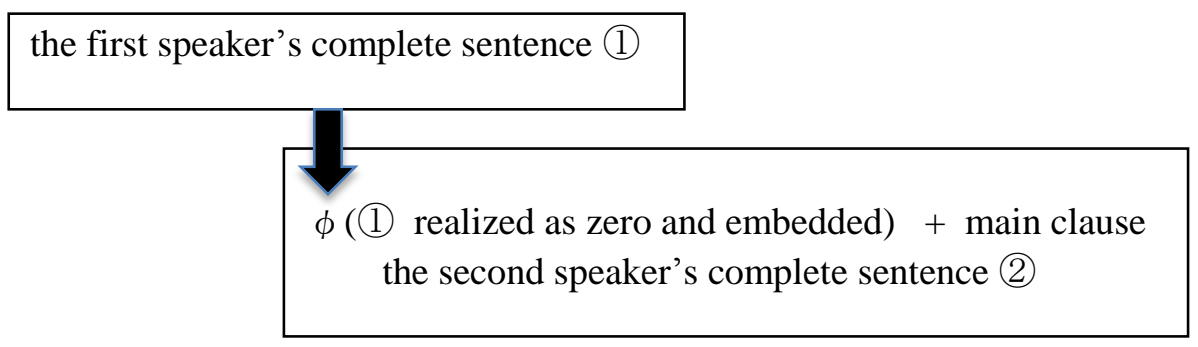

Figure 7. The interlocked structure derived from two independent sentential-level units

The second speaker, therefore, not only takes advantage of the first speaker's component (1) and grammatically converts it into a different role, but also declares syntactic authorship by placing (1) into a subordinate clause in the component (2). On the other hand, the occurrence of (2) 
depends on that of (1). This means that while the speaker of (2) declares equal authorship with the speaker of (1), the occurrence of (2) is not totally independent from the context initiated by (1). In a similar way, from the perspective of action trajectory, (2) offers a new direction by shifting the original frame maintained by (1) to a different one (i.e., shifting from a comment to a quotation), and yet both (1) and (2) are closely bound together in the whole discourse because (2) is an extension of the action instantiated by (1).

Therefore, we argue that co-authored joint utterances present features that go beyond those encapsulated by the current distinction between TCU continuation and new TCUs. They are not like traditional TCU continuations in which increments are added to the host component. They are co-created by two speakers through two independent sentential-level units, and yet their syntactic construction is so tightly-knitted that they are structurally inseparable. Co-authored joint utterances are something speakers produce jointly as if weaving (independent) strands into a single tapestry.

In conversational interaction, two (or more) participants perform dynamic acts through the toand-fro of fragments, retrospective add-ons, interruptions and synchronisms, sudden stops and unexpected silences. Turns are thus constantly extemporaneous. Ford, et al. (1996) and Ford (2004) assert that TCUs are emergent and cannot be pre-defined because conversation involves "the management of simultaneously unfolding facets of action, sound production, gesture, and grammar produced by multiple participants" (Ford, 2004: 27). This view of TCUs undermines, however, the emphasis placed on syntactic structure in making the distinction between TCU continuation and new TCUs, and thus the implicit reliance on syntax in defining TCUs themselves, a point of critique which has been noted by others working in CA (Ford, et al., 1996, 2013).

What is more, there are a lot more significant features at possible points of TCU completion, or TRPs, where the transition from one speaker to another can take place; for example, rules and restrictions when amalgamating two syntactic units into one component, semantic diversities and changes in speaker stance between the participants, and characteristics of the Japanese language which make it possible to create co-authored joint utterances. Therefore, analysing an utterance as either a TCU continuation or two distinct TCUs would "miss building an account of what people are doing in interaction" because various practices, "syntactic, pragmatic prosodic, gestural, can be drawn upon in a wide variety of ways to frame conversational actions as nearing, or not nearing, completion, and thus displaying participants' understanding of whether or not it is someone else's talk" (Ford, et al., 1996: 450).

There is another point to be noted. We assume that in analysing interaction in languages such as Japanese which allow ellipsis in all sorts of places in utterances, we would face more difficulties in distinguishing new TCUs from TCU continuations. Couper-Kuhlen (2012: 298) argues that "in languages where so-called 'zero-arguments' abound, ... TCU continuations and new TCUs would be all the more difficult to distinguish". The examples of co-authored joint utterances we have presented in this paper are indeed just such an example that takes advantage of zero arguments, thereby enabling two sentential-level units to be conjoined. These two independent units are intertwined as if original strands are no longer autonomous strands but woven into a tapestry. It is our contention that in the case of such tightly-knitted phenomena, the distinction between TCU continuation and new TCUs is no longer significant for either participants or analysts. 


\section{Conclusion}

In this paper, we have introduced a type of joint utterance, in which two independent sententiallevel units are conjoined by each sharing a part of the other unit. We call these 'co-authored joint utterances' because both speakers equally control their own utterance; they are not a mere expansion of the host component or completion of the first speaker's incomplete utterance. Syntactically, two independent sentential-level units are amalgamated, and the omitted units (in the second speaker's component) function as the merging point for the whole construction. The co-authored joint utterance is unique due to two sentential-level units being involved and partially merged on the same syntactic plane.

We have also examined the applicability of the distinction between TCU continuation and new TCUs in relation to examples of co-authored joint utterances. We have argued that the construction of co-authored joint utterances goes beyond a matter of 'conversational turns' as examined through the lens of TCUs, because although two participants are involved, the construction is fused and inseparable. It is a jointly interlaced unit for which the distinction between TCU continuation and new TCU no longer appears significant for those participants.

\section{Terms used in morphological gloss}

ACC - accusative case marker

Conj-conjunction

Cop - copula, $d a$ and its conjugated forms

MD - mood marker

Nomi - nominalizer

Pass - passive forms, -reru/-rareru

Past - past tense, $-t a$

Polite - polite forms, masu and desu

Prog - progressive auxiliary

$\mathrm{Q}$ - question marker, $k a$

Quote - quotation from, to and its variations such as -tte, -toiu

$\mathrm{TE}$ - the form which bridges between a verb and an auxiliary

Top - topic marker, wa

\section{Transcription conventions}

$\begin{array}{ll}= & \text { talk 'latched' onto previous speaker's talk } \\ : & \text { stretching of sound } \\ \text { falling intonation } \\ \text { [ ] } & \text { overlapping talk } \\ \text { CAPITALS } & \text { markedly louder talk } \\ \text { (.) } & \text { micro-pause }\end{array}$

Funding acknowledgement: This work was supported by Grant-in-Aid for Scientific Research (KAKENHI), the Ministry of Education, Culture, Sports, Science and Technology, Japan (Grant No. Kiban (C) - 23520537) 


\section{References}

Peter Auer (2007). Why are increments such elusive objects? - an afterthought. Pragmatics, 17: 647-658.

Peter Auer (2009). Projection and minimalistic syntax in interaction. Discourse Processes, 46: 180-205.

Galina B. Bolden (2003). Multiple modalities in collaborative turn sequences. Gesture, 3: 187212.

Ronny Cann, Ruth Kempson and Lutz Marten (2005). The Dynamics of Language. Oxford: Elsevier.

Steven E. Clayman (2013). Turn-constructional units and the transition-relevance place. In Sidnell, J and Stivers, T. (eds.) Handbook of Conversation Analysis. Malden, MA: WileyBlackwell, pp.150-166.

Elizabeth Couper-Kuhlen (2012). Turn continuation and clause combinations. Discourse Processes, 49: 273-299.

Elizabeth Couper-Kuhlen and Ono Tsuyoshi (2007). Incrementing in conversation: a comparison of methods in English, German and Japanese. Pragmatics, 17(4): 513-552.

Cecilia E. Ford (2004). Contingency and units in interaction. Discourse Studies, 6(1): 27-52.

Cecilia E. Ford, Barbara A. Fox, and Sandra A. Thompson (1996). Practices in the construction of turns: the "TCU" revisited. Pragmatics, 6(3): 427-454.

Cecilia E. Ford, Barbara A. Fox, and Sandra A. Thompson (2002). Constituency and the grammar of turn increments. In: Ford, C.E., Fox, B. Aa, and Thompson, S.A. (eds.) The Language of Turn and Sequence. Oxford: Oxford University Press, pp.14-38.

Cecilia E. Ford, Barbara A. Fox, and Sandra A. Thompson (2013). Units and/or action trajectories? - the language of grammatical categories and the language of social action. In: Szczepek, R.B. and Raymond, G. (eds.) Units of Talk - Units of Action. Amsterdam: John Benjamins, pp.13-55.

Barbara A. Fox, Makoto Hayashi, and Robert Jasperson (1996). Resources and repair: A crosslinguistic study of syntax and repair. In: Ochs, E., Schegloff, E.A., and Thompson, S.A. (eds.) Interaction and Grammar. Cambridge: Cambridge University Press, pp.185-237.

Barbara A. Fox, Sandra A. Thompson, Cecilia E. Ford, and Elizabeth Couper-Kuhlen (2013). Conversation analysis and linguistics. In: Sidnell, J. and Stivers, T. (eds.) Handbook of Conversation Analysis. Malden, MA: Wiley-Blackwell, pp.726-740.

Eleni Gregoromichelaki, Ruth Kempson, Matthew Purver, Gregory J. Mills, Ronnie Cann, Wilfried Meyer-Viol, and Patrick G. Healey (2011). Incermentality and intention-recognition in utterance Processing. Dialogue and Discourse, 2(1): 199-233.

Michael Haugh (2010). Co-constructing what is said in interaction. In: Eniko, N.T. and Bibok, K. (eds.) The Role of Data at the Semantics-Pragmatics Interface. Berlin: DeGruyter Mouton, pp.349- 380 .

Makoto Hayashi (1999). Where grammar and interaction meet: a study of co-participant completion in Japanese conversation. Human Studies 22: 475-499.

Makoto Hayashi (2003). Joint Utterance Construction in Japanese Conversation. Amsterdam/Philadelphia: John Benjamins Publishing Company.

Makoto Hayashi and Junko Mori (1998). Co-construction in Japanese revisited: we do"finish each other's sentences". In: Akatsuka, N., Hoji, H., Iwasaki, S., Sohn, S.O., and Strauss, S. (eds.) Japanese/Korean Linguistics, Volume 7. Stanford/California: CSLI Publications, pp.77-93. 
Christine Howes, Matthew Purver, Patrick G. Healey, Gregory J. Mills, and Eleni Gregoromichelaki (2011). On incrementality in dialogue: evidence from compound contributions. Dialogue and Discourse, 2(1): 279-311.

Sally, Jacoby and Elinor Oshs (1995). Co-construction: an introduction. Research on Language and Social Interaction, 28: 171-183.

Talmy Givón (1983). Topic continuity in discourse: an introduction. In: Givón T. (ed.) Topic continuity in Discourse (Typological Studies in Language. Vol. 13). London: John Benjamins Publishing Company, pp.1-14.

John Hinds (1978). Anaphora in Japanese conversation'. In: Hinds J. (ed.) Anaphora in discourse. Edmonton: Linguistic Research Inc., pp.136-179.

John Hinds (1982). Ellipsis in Japanese. Carondale and Edmonton: Linguistic Research Inc.

John Hinds (1983). Topic continuity in Japanese. In: Givón T. (ed.) Topic continuity in discourse. Amsterdam: John Benjamins Publishing Company, pp.43-93.

John Hinds (1984). Topic maintenance in Japanese narratives and Japanese conversational interaction. Discourse Processes 7: 465-482.

Ruth Kempson, Wilfried Meyer-Viol and Dov Gabbay (2001). Dynamic Syntax: The Flow of Language Understanding. Oxford: Blackwell.

Jun-Ju Kim (1999). The co-construction of utterances in Korean in face-to-face conversation between friends. Crossroads of Language, Interaction, and Culture, 1: 61-75.

Ross Krekoski (2012). Causal continuations in Japanese. Discourse Processes, 49: 300-313.

Ritva Laury and Tsuyoshi Ono (2014). The limits of grammar: clause combining in Finnish and Japanese conversation. Pragmatics, 24: 561-592.

Gene Lerner (1991). On the syntax of sentences-in-progress. Language in Society, 20: 441-458.

Gene Lerner (1996). On the 'semi-permeable' character of grammatical units in conversation: conditional entry into the turn space of another speaker. In: Ochs, E., Schegloff, E.A., and Thompson, S.A. (eds.) Interaction and Grammar. Cambridge: Cambridge University Press, pp.238-276

Gene Lerner (2004). On the place of linguistic resources in the organization of talk-in-interaction: Grammar as action in prompting a speaker to elaborate. Research on Language and Social Interaction, 37: 154-184.

Gene Lerner and Tomoyo Takagi (1999). On the place of linguistic resources in the organization of talk-in-interaction: a co-investigation of English and Japanese grammatical practices. Journal of Pragmatics, 31: 49-75.

Anthony J. Liddicoat (2004) The projectability of turn constructional units and the role of prediction in listening. Discourse Studies, 6(4): 449-469.

John Local (2005). On the interactional and phonetic design of collaborative completions. In: Hardcastle, W. and Beck, J. (eds.) Figure of Speech: A Festschrift for John Laver. Mahwah, NJ: Lawrence Erlbaum, pp.263-282.

Kwang-Kwong Luke and Wei Zhang (2007). Retrospective turn continuations in mandarin Chinese conversation. Pragmatics 17: 605-635.

Tsuyoshi Ono and Elizabeth Couper-Kuhlen (2007). Increments in cross-linguistic perspective: introductory remarks. Pragmatics, 17(4): 505-512.

Tsuyoshi Ono and Sandra A. Thompson (1996). Interaction and syntax in the structure of conversational discourse: collaboration, overlap, and syntactic dissociation. In: Hovy, E.H. and Scott, D.R. (eds.) Computational and Conversational Discourse: Burning Issues - A Interdisciplinary Account. Berlin: Springer-Verlag, pp.67-96. 
Massimo Poesio and Hannes Rieser (2010). Completions, coordinations, and alignment in dialogue. Dialogue and Discourse, 1: 1-89.

Matthew Purver, Eleni Gregoromichelaki, Wilfried Meyer-Viol and Ronnie Cann (2010). Splitting the "I" $s$ and crossing the "you" : Context, speech acts and grammar. Proceedings of SemDial (PozDial) 2010: 43-50.

Christoph Rühlemann (2007) Conversation in Context - A Corpus-Driven Approach. London: Continuum.

Harvey Sacks (1992a). Lectures on Conversation (Vol.1). Oxford: Blackwell. Harvey Sacks (1992b). Lectures on Conversation (Vol.2). Oxford: Blackwell.

Harvey Sacks, Emanuel A. Schegloff, and Gail Jefferson (1974). A simplest systematic for the organization of turn-taking for conversation. Language, 50: 696-735.

Emanuel A. Schegloff (1996). Turn organization: one intersection of grammar and interaction. In: Ochs, E., Schegloff, E.A., and Thompson, S.A. (eds.) Interaction and grammar. Cambridge: Cambridge University Press, pp.52 - 133.

Emanuel A. Schegloff (2001). Conversation analysis: a project in process - "increments". Forum Lecture, LSA Linguistic Institute, UC Santa Barbara.

Margaret Selting (2000). The construction of units in conversational talk. Language in Society, 29: 477-517.

Jack Sidnell (2012). Turn-continuation by self and other. Discourse Processes, 49: 314-337.

Jürgen Streeck and Ulrike Hartge (1992). Gestures at the transition place. In: Auer, P. and di Luzio, A. (eds.) The Contextualization of Language. Amsterdam/Philadelphia: John Benjamins Publishing Company, pp.135-157.

Beatrice Szczepek (2006). Prosodic Orientation in English Conversation. Basingstoke: Palgrave Macmillan.

Tanaka Hiroko (1999). Turn-Taking in Japanese Conversation: A Study in Grammar and Interaction. Amsterdam/Philadelphia: John Benjamins Publishing Company.

Tanaka Hiroko (2000). Turn-projection in Japanese talk in interaction. Research on Language and Social Interaction 33: 1-38. 